\section{Initial Localization by Set Inversion}

Dominique Meizel, Olivier Lévêque, Luc Jaulin, and Eric Walter

Abstract-In this paper, initial localization problems are solved by using set-membership estimation. The method can be used with any robot and any kind of sensor(s), provided that a computable model of the environment/sensor interaction is available. With a pedagogical aim in mind, it is detailed in the case of the localization of a vehicle from range measurements in a polygonal environment. Salient properties of the method are as follows. First, it does not need any explicit management of matching hypotheses. Second, it is able to deal with ambiguous situations where several radically different vehicle configurations are consistent with the measurements. Third, it can be made robust to outliers. Fourth, it can deal with nonlinear observation models without any approximation. Fifth, the result is guaranteed in the sense that no configuration consistent with the data and the hypotheses can be missed.

Index Terms-Guaranteed estimation, interval analysis, localization, mobile robotics, set-membership estimation.

\section{INTRODUCTION}

A robot is a machine that has been designed to interact with the real world in order to perform a given set of tasks. In the case of a mobile robot, reaching the right pose (or configuration) with respect to the goal of the mission is an important subtask; this is why localization is still an active research topic. The choice of the reference frame as well as the formal representation of the workspace depend on the task to be performed.

\section{A. Workspace Representation}

When the mission is just the exploration of an unknown environment while avoiding collisions, an occupancy grid [1], [2] is an effective means to represent either the temporary obstacles or the landmarks that structure the environment. In such a representation, a confidence coefficient associated with each cell quantifies the belief in the fact that the cell is occupied. During the exploration of the environment, the local maps are integrated [3] and the occupancy coefficients are updated as the robot moves. Even if there is an ongoing effort to design more effective algorithms, such a cell-based model is intrinsically greedy in memory space, which is the price paid to manage uncertainty in the knowledge of the environment or in the specification of the task

When the task is clearly defined, using analytical expressions to express the relations between the mobile and the items of the environment saves memory space and yields localization methods that are potentially less time consuming than cell-based representations [4]. For instance, analytical representations are most frequently used in sensor-referenced control where the task to be performed is implemented by output feedback regulation algorithms. Common sense, nevertheless, suggests supplementing the basic feedback motion

Manuscript received July 16, 2001; revised May 17, 2002. This paper was recommended for publication by Associate Editor J. Leonard and Editor S. Hutchinson upon evaluation of the reviewers' comments. This work has been sponsored by the Picardie region within the DIVA pole for research and technological transfer.

D. Meizel is with UTC/HEUDIASYC, 60205 Compiegne Cedex, France (e-mail: Dominique.Meizel@hds.utc.fr).

O. Lévêque was with UTC/HEUDIASYC, 60205 Compiegne Cedex, France. He is now with Globalware, 75017 Paris, France.

L. Jaulin is with the Laboratoire d'Ingénierie des Systèmes Automatisés (LISA), 49045 Angers, France.

E. Walter is with the Laboratoire de Signaux et Systèmes (LSS), CNRS-Supelec-Université Paris-Sud, 91192, Gif sur Yvette, France.

Digital Object Identifier 10.1109/TRA.2002.805664 generators with supervision processes based on the updating of a complete pose estimate. Output-feedback regulation can then be implemented by feeding back the state estimate, which includes the pose.

\section{B. State Estimation}

Extended Kalman filtering (EKF) is ubiquitous in state estimation for dynamic processes. Applied to the localization of mobile robots, it provides an integrated solution for the tracking of initial estimates of the pose [5]. In this technique, the covariance matrix updated along with the state estimate quantifies the estimation inaccuracy [6] and allows validation gates [7] to be defined and used for the recognition of landmarks. When there are several possible initial estimates, multitarget tracking [8] can be used to extend the original localization mechanism [9]. A finite set of good initial estimates remains, nevertheless, needed.

Set-membership estimation is an alternative solution for the estimation of the state of dynamic processes [10], [11]. This methodology characterizes the set of all states consistent with the available measurements as interpreted by an observation model. Compare with EKF-based solutions, which consist of point estimates and local covariance matrices. The first set-membership methods introduced in robotics [12], [13] were based on a linearization of the equations, and used ellipsoidal domains to enclose the solution sets; this choice corresponded to the availability and convenience of ellipsoidal algorithms together with the possibility of comparing the solutions with those based on EKF. Later on, an exact polyhedral description has been used with more accurate results [14]. An important property of set-membership estimation is its greater ability to deal with short data sets compared to the methods relying upon the usual Gaussian hypothesis [15]. This is especially useful during initialization.

\section{Initial Localization}

The search for initial pose estimates has been the subject of a renewed interest during the last four years. Drumheller [16] introduced the concept of an interpretation tree in the context of localization, and designed a strategy to prune this interpretation tree to limit complexity. More recently, Crowley et al. [17] used pattern recognition to match dense measurements with a set of landmarks in the map of the environment via a principal component representation of the measurements. Markovian approaches [18] consider localization as the computation of a probability distribution over the free configuration space. This approach allows a total lack of initial knowledge about the robot location to be represented by a uniform initial distribution. Each new measurement, possibly obtained after a robot displacement, is interpreted in a statistical framework and used to update the probability distribution of the location. This technique can give rise to multimodal distributions when the perception of the environment is symmetrical, thus making it possible to deal with ambiguous situations without the need of prior estimates. Technically, the probability distribution is computed on a grid representation of the free configuration space. This allows the use of a map learned after an exploration phase with the correlative disadvantage of the large amount of memory and computation time needed. To overcome this problem of complexity, methods with adaptive step sizes have been proposed, based on the use of octrees [19] or on Monte-Carlo sampling [20]. The navigation of a robot in a totally or partially unknown environment is a typical application of this type of method. In [21], this Markovian approach is used, together with an optimization procedure that estimates the most probable cell inside the updated grid; superimposing interpolation functions allows the precision of the estimate to be increased. 
With the same kind of data obtained from an omnidirectional vision sensor, it is proposed in [22] to partition the free space iteratively. The two-dimensional (2-D) workspace is structured by characteristic points, and the measurements are the angles under which couples of characteristic points are seen. The inaccuracy of the measurement is expressed by bounds on the errors.

This paper extends some results developed in [23] and presented in [24]. It is focused upon the initial localization problem and defines a general theoretic framework, which can be used with any kind of sensors and landmarks. The only assumptions are the availability of 1) a sufficiently representative map, and 2) a computable model of the interaction between the sensors and the environment (both constitute an observation simulator). Assumption 1) is not restrictive, since the map is part of the task definition. The proposed technique uses a set-inversion algorithm [25] based on interval analysis to find an initial solution set compatible with the available measurements and an environment model consisting of a collection of landmarks.

This paper is organized as follows. In Section II, localization will be interpreted as the inverse operation of the simulation of onboard measurements, while the observation relations are shown to be combinations of equalities and inequalities. Section III recalls the basics of the set inversion via interval analysis (SIVIA) algorithm, which will be used to perform the inversion of a simulator that predicts the sensor data for any given configuration of the robot. Section IV illustrates the method on two simulated examples, the latter showing how the method is able to deal with ambiguous situations. An adaptation of the method that takes outliers into account is illustrated using data obtained with an actual robot.

\section{BOUNDED-ERROR FORMULATION OF OBSERVATIONS}

Let $\mathcal{W}$ be a reference frame tied to the workspace, i.e., the part of the environment where the mission of the robot is defined, and $\mathcal{M}$ be a reference frame tied to the vehicle. Localization is understood as the action performed to estimate the pose $\mathbf{q}$ which specifies the transformation from $\mathcal{M}$ to $\mathcal{W}$. Consider a simple vehicle as shown in Fig. 1. q can be defined as

$$
\mathbf{q}=\left[x_{C}, x_{C}, \theta\right]^{T} \text {. }
$$

The vehicle is equipped with external sensors that provide information about the progress and completion of its mission; telemeters and artificial vision systems are typical examples of such sensors. Each sensor performs an interaction between the vehicle and at least one landmark ${ }^{\mathcal{W}} \mathcal{S}$ of the environment. ${ }^{1}$ This relation is generally written as an observation equation

$$
{ }^{\mathcal{W}} \mathbf{z}={ }^{\mathcal{M}} \mathbf{h}\left(\mathbf{q},{ }^{\mathcal{W}} \mathcal{S}\right)
$$

In the sequel, the simple academic example of a telemetric measurement will show the relevance of completing those equalities with inequalities, leading to the generalized formulation of an interaction

$$
\left\{\begin{array}{l}
\mathcal{W}_{\mathbf{z}}={ }^{\mathcal{M}} h\left(\mathbf{q}^{{ }^{\mathcal{W}}} \mathcal{S}\right) \\
0 \leq \mathcal{M}_{g}\left(\mathbf{q},{ }^{\mathcal{W}} \mathcal{T}\right) .
\end{array}\right.
$$

\section{A. Ideal Telemetric Measurement With Respect to a Facet}

Consider a perfectly accurate telemeter in an ideally Lambertian environment (Fig. 2). The telemeter is installed on the vehicle at the point

\footnotetext{
${ }^{1}$ As usual in mechanics, ${ }^{\mathcal{F}} x$ denotes the coordinates of the vector $x$ in the reference frame $\mathcal{F}$
}

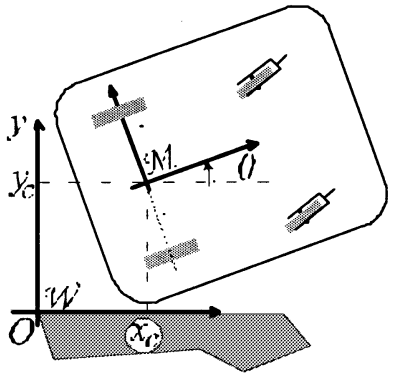

Fig. 1. Pose of a vehicle.

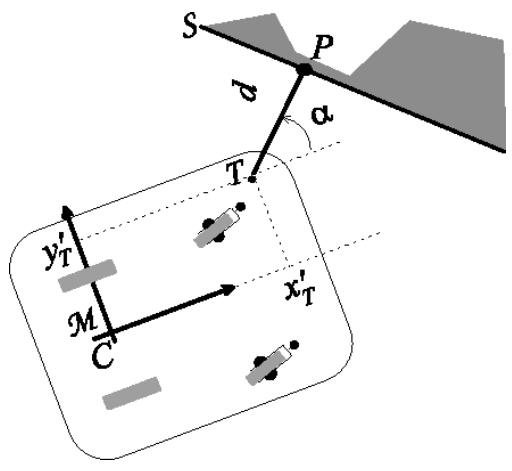

Fig. 2. Ideal telemetric measurement.

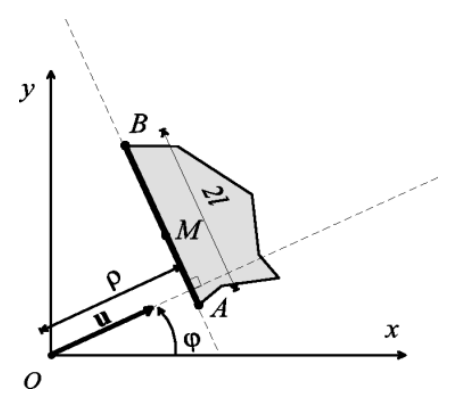

Fig. 3. Attributes of a facet landmark.

$T$ with coordinates ${ }^{\mathcal{M}}\left[x_{T}^{\prime}, y_{T}^{\prime}\right]^{T}$; it emits and receives a unidirectional wave in the direction $\alpha^{\prime}$. When the sensor provides a distance $d$, this means that there exists an obstacle point $P$ described indifferently in $\mathcal{M}$ by (4), or in $\mathcal{W}$ by (5) (in the sequel, $R(\varphi)(3)$ denotes a rotation of angle $\varphi$ )

$$
\begin{aligned}
R(\varphi) & =\left[\begin{array}{cc}
\cos (\varphi) & -\sin (\varphi) \\
\sin (\varphi) & \cos (\varphi)
\end{array}\right] \\
\mathcal{M} \overrightarrow{C P} & =\left[\begin{array}{ll}
x_{T}^{\prime} & y_{T}^{\prime}
\end{array}\right]^{T}+R\left(\alpha^{\prime}\right) \cdot\left[\begin{array}{ll}
d & 0
\end{array}\right]^{T} \\
\mathcal{W} \overrightarrow{O P} & =\left[\begin{array}{ll}
x_{C} & y_{C}
\end{array}\right]^{T}+R(\theta) \cdot{ }^{\mathcal{M}} \overrightarrow{C P} .
\end{aligned}
$$

Consider a facet ${ }^{\mathcal{W}} \overrightarrow{A B}$ described as displayed in Fig. 3. $A$ and $B$ are its vertices, $M$ its midpoint, $l$ its half length, ${ }^{\mathcal{W}} \vec{u}=[\cos (\varphi), \sin (\varphi)]^{T}$ the normal vector directed toward the inner part of the facet, and $\rho$ the distance between the origin $O$ of $\mathcal{W}$ and the straight line $(A, B)$ that supports $\overrightarrow{A B}$.

The fact that a given point $P$ belongs to $\mathcal{W} \overrightarrow{A B}$ is expressed by the three following facts.

1) $P$ belongs to the straight line $(A, B)$ as described by the equality (6).

2) $P$ lies between $A$ and $B$ as stated by the inequality (7). 
3) The telemeter $T$ is in the outer part of the facet as expressed by the inequality (8).

$$
\begin{aligned}
\langle\overrightarrow{O P}, \vec{u}\rangle & =\rho \\
\langle\overrightarrow{M P}, \overrightarrow{M A}\rangle & \leq l^{2} \\
\operatorname{det}(\overrightarrow{T A}, \overrightarrow{T B}) & \geq 0 .
\end{aligned}
$$

Those relations, together with the definition of $\overrightarrow{O P}(5)$ that depends on the pose $\mathbf{q}$, is an instance of the general form (2).

\section{B. Less Ideal Telemetric Measurement With Respect to a Facet}

Let us now take into account the inaccuracy of telemetric measurements by considering 1) the dispersion of the emitted wave inside a cone and 2) the necessarily limited relative precision.

From a geometrical point of view, it means that, when a measurement $\left(d, \alpha^{\prime}\right)$ is available, the detected point should no longer correspond to a unique point, as in (4), but rather to a crown sector ${ }^{\mathcal{M}} \mathcal{C S}\left(d, \alpha^{\prime}\right)$

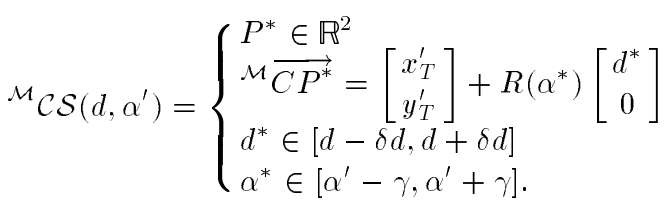

The induced localization relation is then translated into a system of inequalities expressing that there exists at least one point $P^{*}$ satisfying both of the two following relations: 1) $P^{*}$ belongs to the crown sector $\mathcal{W}_{\mathcal{C S}}\left(d, \alpha^{\prime}\right)$ tied to the sensor and expressed by relations (5) and (9), and 2) $P^{*}$ belongs some facet $\mathcal{W} \overrightarrow{A B}$, i.e., $P^{*}$ satisfies (6) and (7), and the sensor lies on the reflecting side of $\mathcal{W} \overrightarrow{A B}$ as expressed by (8).

These last relations instantiate the general form (2) where the inequalities (10) are directly linked with the measured data $\left(d, \alpha^{\prime}\right)$, whereas the equalities constraints (11) depend on the pose $\left(x_{C}, y_{C}, \theta\right)$.

Both define a subset $\mathcal{S}\left(d, \alpha^{\prime}\right)$ of the configuration set $\mathbb{R}^{2} \times[-\pi,+\pi]$. The practical computation of the intersection $\cup_{i=1}^{n} \mathcal{S}\left(d_{i}, \alpha_{i}^{\prime}\right)$ of the subsets $\mathcal{S}\left(d_{i}, \alpha_{i}^{\prime}\right)$ corresponding to $n$ different measurements obtained at the same pose is the principle of the method developed in the sequel

$$
\begin{aligned}
& \left\{\begin{array}{l}
d^{*} \in[d-\delta d, d+\delta d] \\
\alpha^{*} \in\left[\alpha^{\prime}-\gamma, \alpha^{\prime}+\gamma\right] \\
l^{2} \geq\left\langle\overrightarrow{M P^{*}}, \overrightarrow{M A}\right\rangle \\
0 \leq \operatorname{det}(\overrightarrow{T A}, \overrightarrow{T B})
\end{array}\right. \\
& \left\{\begin{array}{l}
\mathcal{W} \overrightarrow{O P^{*}}=x_{C} \quad y_{C}^{T}+R(\theta) \cdot^{\mathcal{M}} \overrightarrow{C P^{*}} \\
\mathcal{M} \overrightarrow{C P^{*}}=x_{T}^{\prime} \\
\rho=\left\langle\overrightarrow{O P^{*}}, \vec{u}\right\rangle
\end{array} y_{T}^{T}+R\left(\alpha^{\prime}\right) \cdot d 0^{T}\right.
\end{aligned}
$$

\section{INVERSE PROBLEM OF SiMULATION}

Consider a vehicle equipped with $n$ sensors and a description of the workspace by a set of $m$ facets. ${ }^{\mathcal{W}} \mathcal{S}=\left\{{ }^{\mathcal{W}} \mathcal{S}_{i}, i=1, \ldots, m\right\}$ (some of the facets define the reference frame $\mathcal{W}$ that is significant with respect to the mission). A deterministic observation simulator is a function s (12) computing the measurements that should be reported by the $n$ sensors if the configuration of the vehicle were $\mathbf{q}$, assuming that the models of the sensors and of the environment are correct. Localization can then be defined as the reciprocal operation of simulation. Assume that the inaccurate experimental data are defined as a vector of range intervals (13) and the set $\mathcal{Q}$ of feasible poses is defined as the reciprocal image of $\mathbf{r}^{\mathrm{I}}$ by $\mathbf{s}$

$$
\begin{aligned}
\mathbf{q} \stackrel{\mathbf{s}}{\longmapsto} & {\left[\begin{array}{llll}
d_{1}, & d_{2} & \ldots & d_{n}
\end{array}\right]^{T} } \\
\mathbf{r}^{\mathbf{I}}= & {\left[\begin{array}{llll}
{\left[r_{1}^{-}, r_{1}^{+}\right]} & {\left[r_{2}^{-}, r_{2}^{+}\right]} & \ldots & {\left[r_{n}^{-}, r_{n}^{+}\right]}
\end{array}\right]^{T} . }
\end{aligned}
$$

The outer approximation of $\mathcal{Q}$ will be computed by the SIVIA algorithm. Section III-A is devoted to introducing the minimal interval analysis concepts that are necessary to understand the use of SIVIA in this practical context. More information can be found in [26].

\section{A. Interval Analysis and Set Inversion}

Interval analysis has been originally [27] developed to analyze the numerical problems caused by the finite length of the representation of numbers in computers. It is now a mature technique for which computation tools are readily available. Scalar intervals are basic objects when modeling measurement inaccuracy in the bounded-error framework, as illustrated by (10). When $N$ interval range measurements are available, they form a vector of intervals, or more briefly, a box.

Concerning real intervals, the following notation is used:

- $\mathbb{R}$ is the set of scalar real intervals $\left\{x^{I} \in \mathbb{R} \Leftrightarrow x^{I}=\right.$ $\left.\left[x^{-}, x^{+}\right] \subset \mathbb{R}\right\}$;

- $\mathbb{R}^{N}$ is the set of $N$-dimensional boxes $\left\{\mathbf{x}^{I} \in \mathbb{\mathbb { R } ^ { N }} \Leftrightarrow \mathbf{x}^{I}=\right.$ $\left.\left[\mathbf{x}^{-}, \mathbf{x}^{+}\right] \subset \mathbb{\mathbb { R } ^ { N }}\right\}$; such that

$$
\left\{\mathbf{x} \in \mathbf{x}^{I}\right\} \Longleftrightarrow\left\{\forall i \in\{1,2, \ldots, N\}, \mathbf{x}_{i}^{-} \leq \mathbf{x}_{i} \leq \mathbf{x}_{i}^{+}\right\} \text {. }
$$

An inclusion function $\mathbf{f}^{\mathbf{I}}$ of a function $\mathbf{f}$ from $\mathbb{R}^{N}$ to $\mathbb{R}^{M}$ is an application from $\mathbb{R}^{N}$ to $\mathbb{R}^{M}$, such that for any box $\mathbf{x}^{\mathbf{I}}$, the box $\mathbf{f}^{\mathbf{I}}\left(\mathbf{x}^{\mathbf{I}}\right)$ includes $\mathbf{f}\left(\mathbf{x}^{\mathbf{I}}\right)$. Inclusion functions are used to obtain implementable inclusion tests. To check directly whether the image of a box $\mathbf{x}^{\mathrm{I}}$ is inside a feasible domain may be impossible, since the set $\mathbf{f}\left(\mathbf{x}^{\mathbf{I}}\right)$ is not explicitly computable except in very special cases. On the other hand, if an inclusion function $\mathbf{f}^{\mathrm{I}}$ can be designed, it becomes easy to test whether $\mathbf{f}^{\mathbf{I}}\left(\mathbf{x}^{\mathbf{I}}\right)$, which is a box, is included in the feasible set, which is often also a box. This test gives sufficient feasibility conditions that are more or less conservative, depending on how the inclusion function $\mathbf{f}^{\mathrm{I}}$ has been derived from the original one $\mathbf{f}$.

\section{B. SIVIA Algorithm}

The problem to be solved is the guaranteed estimation of the reciprocal image $\mathcal{Q}$ of a feasible measurement set $\mathbf{r}^{\mathrm{I}}$ (13) by the simulator function $\mathbf{s}$ described by (12). In this context, the inputs of SIVIA are an initial box $\mathbf{q}_{0}^{I}$ that should contain all configurations of interest, an inclusion function $\mathbf{s}^{I}$ for the simulator $\mathbf{s}$, and a precision parameter $\varepsilon$.

A box $q^{I}$ will be classified as:

- feasible if $\mathbf{s}^{\mathbf{I}}\left(q^{I}\right) \subset \mathbf{r}^{\mathbf{I}}$;

- infeasible if $\mathbf{s}^{\mathbf{I}}\left(q^{I}\right) \cap \mathbf{r}^{\mathbf{I}}=\varnothing$;

- indeterminate in the other cases (see Fig. 4).

SIVIA is a branch-and-bound algorithm. It iteratively builds three sets $\mathcal{L}_{\text {int }}, \mathcal{L}_{\text {ext }}$, and $\mathcal{L}_{\text {ind }}$, described as lists of nonoverlapping boxes. At any step of the algorithm, all boxes in $\mathcal{L}_{\text {int }}$ are feasible, all those in $\mathcal{L}_{\text {ext }}$ are infeasible, and $\mathcal{L}_{\text {ind }}$ contains all those that are indeterminate. Initially, $\mathcal{L}_{\text {int }}=\emptyset, \mathcal{L}_{\text {ext }}=\emptyset$, and $\mathcal{L}_{\text {ind }}=\mathbf{q}_{0}^{I}$.

An iteration of the algorithm consists of the following.

1. Removing a box from $\mathcal{L}_{\text {ind }}$ such that its width is greater than $\varepsilon$ (if no such box exists, the algorithm ends and returns $\mathcal{L}_{\text {int }}$ and $\mathcal{L}_{\text {ind }}$ ) . 


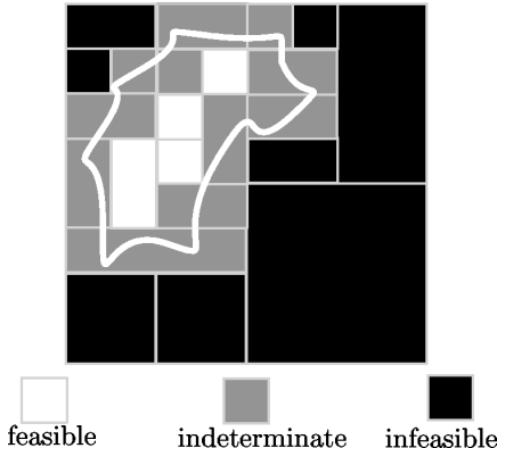

Fig. 4. Bracketing the solution set of $\{s(x) \leq 0\}$ (the interior of the curve) between an inner and an outer approximation: principle.

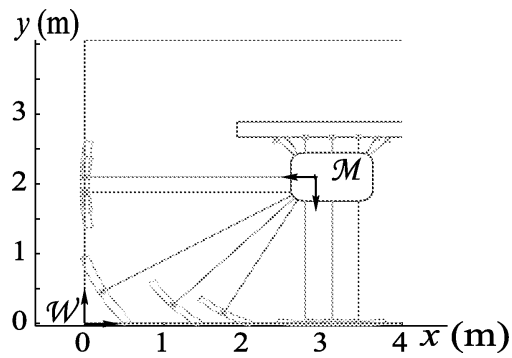

Fig. 5. Nonsymmetrical environment.

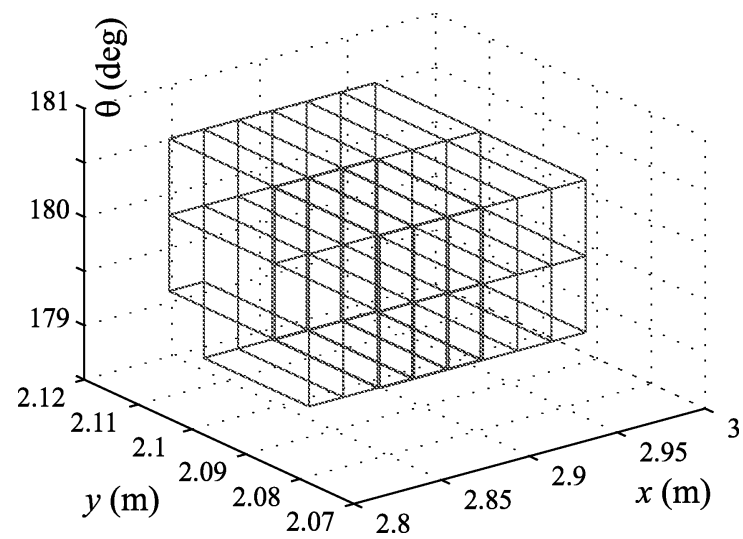

Fig. 6. Outer approximation of the solution set.

2. Appending this box to $\mathcal{L}_{\text {int }}$ if it can be proved to be feasible, or to $\mathcal{L}_{\text {ext }}$ if it turns out to be infeasible. Otherwise, it is indeterminate; it is then cut into two subboxes across one of its principal directions, and these subboxes are appended to $\mathcal{L}_{\text {ind }}$.

Upon completion of the algorithm, the solution set is bracketed between $\mathcal{L}_{\text {int }}$ and $\mathcal{L}_{\text {int }} \cup \mathcal{L}_{\text {ind }}$, and the width of any box in $\mathcal{L}_{\text {ind }}$ is smaller than the precision parameter $\varepsilon$. This algorithm was first presented in [25] and [28] where its complexity and convergence have been studied.

\section{LOCALIZATION BY SET INVERSION}

\section{A. Illustrative Simulated Examples}

The simulated examples considered in this section illustrate the application of the method and its ability to deal with ambiguous situations resulting from local symmetries in the environment. All simulations are

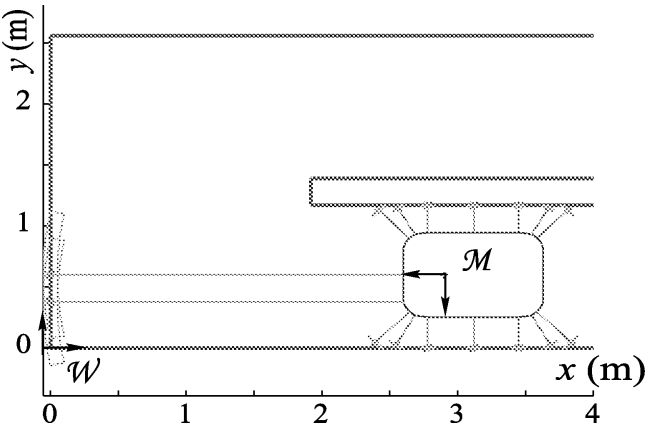

Fig. 7. Symmetrical environment (the widths of the two corridors are equal).

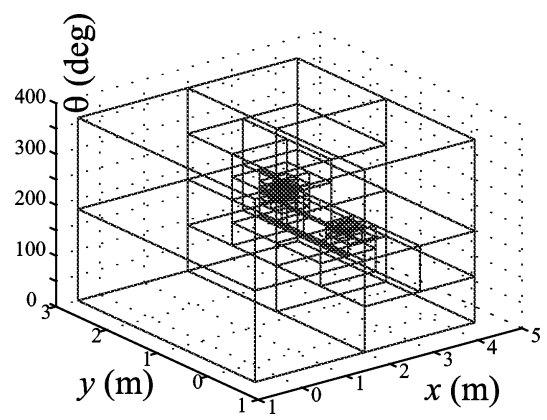

Fig. 8. Iterative decomposition of the initial search domain.

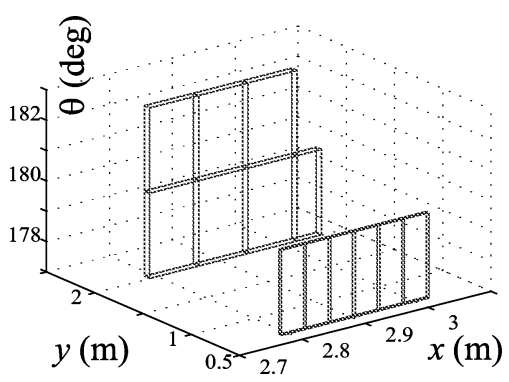

Fig. 9. Outer approximation of the solution set with two disconnected subsets.

performed using realistic values to quantify measurement inaccuracy: $\{|\delta d / d| \leq 2 \%\}$ for range and $\left\{\gamma=10^{\circ}\right\}$ for angular dispersion.

First, consider the simple situation displayed in Fig. 5, where the crown sectors represent the inaccuracy of the measurements. The initial search domain is again taken as $\mathbf{q}_{0}^{I}=\left[[-0.5 \mathrm{~m}, 4.5 \mathrm{~m}],[-0.5 \mathrm{~m}, 3 \mathrm{~m}],\left[0,360^{\circ}\right]\right] ;$ it is iteratively split by SIVIA used with a precision parameter $\varepsilon=0.02 \mathrm{~m}$. The resulting outer approximation of the feasible configuration set is described by Fig. 6 .

Consider now the example shown in Fig. 7, where the widths of the two corridors are equal. SIVIA is applied in the same conditions, and it explores the initial search domain $\mathbf{q}_{0}^{I}$ as shown in Fig. 8. Upon completion of the algorithm, inner and outer approximations of the feasible configuration domain are obtained, which both consist of two disconnected sets (Fig. 9). It turns out that the data are interpretable with two radically different matching hypotheses, the one that corresponds to the simulated situation (Fig. 7), but also the alternative matching hypothesis illustrated in Fig. 10, which was not necessarily predicted, but would give the same measurements.

Notice that the method does not require any explicit management of the matching between the sets of measurements and landmarks, this matching is a by-product of the method, even in the complicated case of ambiguous environments. 


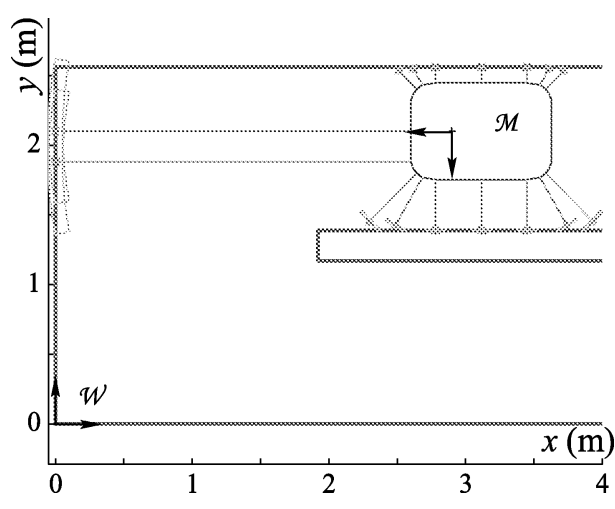

Fig. 10. Alternative matching hypothesis.

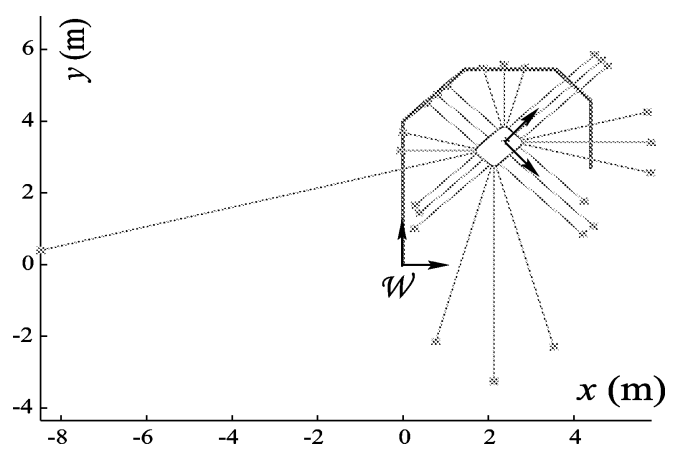

Fig. 11. Actual range data; some aligned impact points are generated by unmapped steps and the longest measured distances.

\section{B. Actual Data and Outliers Management}

When considering real data as, for instance, the ones displayed in Fig. 11 obtained when the robot was in a hall, some range data obviously cannot be associated with any landmark in the map because of the failure of one sensor and of the presence of elements that are not mentioned in the map. The facets $\left\{{ }^{\mathcal{W}} \mathcal{S}_{i} ; i=1 \ldots 5\right\}$ that constitute a description of the workspace have been superimposed on Fig. 11.

One possibility for allowing the presence of outliers in the measurements is to relax the conditions for a box to be feasible when using SIVIA (Section III-B). We can accept $n_{0}$ outliers if the test $\left\{\mathbf{s}^{\mathbf{I}}\left(\mathbf{q}^{\mathbf{I}}\right) \subset \mathbf{r}^{\mathbf{I}}\right\}$ is replaced by the fact that $\left(n-n_{0}\right)$ components of $\mathbf{s}^{I}\left(q^{I}\right)$ (which are scalar intervals) are included in the corresponding components of $\mathbf{r}^{I}$. No other change is necessary.

Consider the experimental data of Fig. 11. If we admit up to $n_{0}=$ 16 outliers and only $n-n_{0}=8$ significant measurements, applying SIVIA with a precision parameter $\varepsilon=0.02 \mathrm{~m}$ and an initial search domain $\mathbf{q}_{0}^{I}=[-0.5 \mathrm{~m}, 5 \mathrm{~m}] \times[-0.5 \mathrm{~m}, 6 \mathrm{~m}] \times\left[0,360^{\circ}\right]$ yields the result shown in Fig. 12. This takes $12 \mathrm{~s}$ using a Pentium 600.

\section{CONCLUSION}

The initial localization problem has been formulated as the inverse operation of the simulation of measurements. The SIVIA algorithm, based on interval computation, provides a systematic, efficient, and general solution to the estimation problem when a model is available to compute the measurements expected for a given configuration of the robot. Set-membership estimation is the theoretical framework of the method, and the inaccuracy of the measurements is expressed in terms of bounds on the possible errors. This set-theoretic approach enables one to use inequalities and not only equations in the observation models. It has also the advantage of not requiring a large number of data, explicitly or implicitly. This is important when measurements are

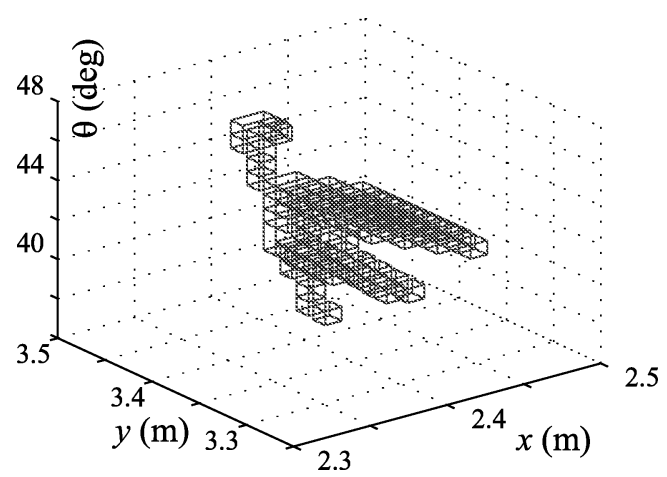

Fig. 12. Outer approximation of the solution set (actual range data).

scarce or when there are many outliers. When the observation model (including the error bounds) is valid, this method gives a guaranteed result in the sense that the true solution must belong to the sets computed. The theoretical simplicity and rigorousness of the method qualify it as a convenient framework for data fusion. The simulation model incorporates descriptions for both the environment and the sensors. The unavoidable differences between the model and the actual system are managed in two ways: lack of accuracy is dealt with by specifying error bounds, uncertainty caused by failures in some sensors or in the simulation model is taken into account by tolerating a prespecified maximum number of outliers. Using fuzzy sets in place of usual sets, in the direction proposed by [29], would be an alternative strategy to take data uncertainty into account.

The computational complexity of the algorithm is bilinear in the number of sensors and in the number of environment items that constitute the map; however, it is exponential in the dimension of the configuration to be estimated. The approach is, thus, practicable for a problem of vehicle localization, where this dimension is three.

Moreover, the algorithm is an "anytime algorithm," which means that each iteration of SIVIA yields a guaranteed outer approximation of the solution set. This outer approximation is a valuable search domain to start a new estimation when new data become available.

A remarkable feature of the approach lies in the fact that, when measurements are ambiguous because of symmetries in the environment, the estimate of the configuration domain may be disconnected, each connected component corresponding to a specific matching hypothesis.

As all configurations in the solution set are equally candidates to be the true one, the strategy to move the robot under this knowledge should minimize a risk criterion rather than maximize an observability criterion. This corresponds to worst-case risk management, as opposed to probabilistic risk management performed if the estimated probability distribution of the localization was used. The solution set will degenerate to a single connected subset only if a subsequent measurement eliminates the ambiguity of the situation. This can be done either by using a more classical multihypotheses localization tracker based upon multitarget tracking, or by integrating the robot motion with the present method. Such a development is done in the framework of the design of set-membership state observers. For first results in the latter direction, see [26] and [30].

\section{REFERENCES}

[1] A. Elfes, "Using occupancy grids for mobile robots perception and navigation," IEEE Computer, pp. 46-57, June 1989.

[2] A. Schultz and W. Adams, "Continuous localization using evidence grids," in Proc. IEEE Int. Conf. Robotics and Automation (ICRA'98), Leuven, Belgium, May 1998, pp. 2833-2839.

[3] — , "Unifying exploration, localization, navigation, and planning through a common representation," in Proc. IEEE Int. Conf. Robotics and Automation (ICRA'99), Detroit, MI, May 1999, pp. 2651-2658. 
[4] B. Schiele and J. Crowley, "A comparison of position estimation techniques using occupancy grids," IEEE Trans. Robot. Automat., vol. 16, pp. 1628-1634, Apr. 1994.

[5] J. Crowley, "World modeling and position estimation for a mobile robot using ultrasonic ranging," in Proc. IEEE Int. Conf. Robotics and Automation (ICRA'89), Scottsdale, AZ, 1989, pp. 674-680.

[6] J. Castellanos, J. Monteil, J. Neira, and J. Tardos, "The smap: A probabilistic framework for simultaneous localization and map building," IEEE Trans. Robot. Automat., vol. 15, pp. 948-952, Oct. 1999.

[7] J. Leonard and H. F. Durrant-Whyte, "Mobile robot localization by tracking geometric beacons," IEEE Trans. Robot. Automat., vol. 7, pp. 376-382, June 1991.

[8] Y. Bar-Shalom and T. E. Fortmann, Tracking and Data Association. Boston, MA: Academic, 1988.

[9] D. Maksarov and H. Durrant-Whyte, "Mobile vehicle navigation in unknown environments: A multiple hypothesis approach," in Proc. IEE Control Theory Applications Conf., July 1995, pp. 385-400.

[10] F. C. Schweppe, Uncertain Dynamic Systems. Englewood Cliffs, NJ: Prentice-Hall, 1973.

[11] M. Milanese, J. Norton, H. Piet-Lahanier, and E. Walter, Bounding Approaches to System Identification. New York: Plenum, 1996.

[12] A. Preciado, D. Meizel, A. Segovia, and M. Rombaut, "Fusion of multisensor data: A geometric approach," in Proc. IEEE Int. Conf. Robotics and Automation (ICRA'91), vol. 3, Sacramento, CA, 1991, pp. 2806-2811.

[13] A. Sabater and F. Thomas, "Set-membership approach to the propagation of uncertain geometric information," in Proc. IEEE Int. Conf. Robotics and Automation (ICRA'91), vol. 3, Sacramento, CA, 1991, pp. 2718-2723.

[14] E. Halbwachs and D. Meizel, "Multiple hypotheses generation for vehicle localization," in Proc. European Control Conf. (ECC'97), Louvain, Belgium, 1997, on CD-ROM.

[15] D. Meizel, A. Preciado-Ruiz, and E. Halbwachs, "Estimation of a mobile robot localization: Geometric approaches," in Bounding Approaches to System Identification, M. Milanese, J. Norton, H. Piet-Lahanier, and E. Walter, Eds. New York: Plenum, 1996, pp. 463-489.

[16] M. Drumheller, "Mobile robot localization using sonar," IEEE Trans. Pattern Anal. Machine Intell., vol. PAMI-9, Feb. 1987.

[17] J. Crowley, F. Wallner, and B. Schiele, "Position estimation using principal components of range data," in Proc. IEEE Int. Conf. Robotics and Automation (ICRA'98), Leuven, Belgium, 1998, pp. 3121-3128.

[18] K. Konolige and K. Chou, "Markov localization using correlation," in Proc. Int. Joint Conf. Artificial Intelligence (IJCAI), 1999, pp. 1154-1159.

[19] A. Burgard, D. Derr, D. Fox, and A. B. Cremers, "Integrating global position estimation and position tracking for mobile robots: The dynamic Markov localization approach," in Proc. IROS Conf., Victoria, BC, Canada, Oct. 1998, pp. 730-735.

[20] F. Dellaert, D. Fox, W. Burgard, and S. Thrun, "Monte-Carlo localization for mobile robots," in Proc. IEEE Int. Conf. Robotics and Automation (ICRA'99), Detroit, MI, 1999, pp. 1322-1328.

[21] C. Olson, "Probabilistic self-localization for mobile robots," IEEE Trans. Robot. Automat., vol. 16, pp. 55-66, Feb. 2000.

[22] E. M. Mouaddib and B. Marhic, "Geometrical matching for mobile robot localization," IEEE Trans. Robot. Automat., vol. 16, pp. 542-552, Oct. 2000.

[23] O. Lévêque, "Méthodes ensemblistes pour la localization de véhicules," $\mathrm{Ph} . \mathrm{D}$. dissertation, Technol. Univ. Compiègne, Compiègne, France, 1998.

[24] O. Lévêque, L. Jaulin, D. Meizel, and E. Walter, "Vehicle localization from inaccurate telemetric data: A set-inversion approach," in Proc. 5th Symp. Robot Control (SYROCO'97), vol. 1, Sept. 1997, pp. 179-186.

[25] L. Jaulin and E. Walter, "Set inversion via interval analysis for nonlinear bounded-error estimation," Automatica, vol. 29, no. 4, pp. 1053-1064, 1993.

[26] L. Jaulin, M. Kieffer, O. Didrit, and E. Walter, Applied Interval Analysis. London, U.K.: Springer-Verlag, 2001.

[27] R. Moore, Methods and Applications of Interval Analysis. Philadelphia, PA: Soc. for Ind. Appl. Math., 1979.

[28] L. Jaulin and E. Walter, "Guaranteed nonlinear parameter estimation from bounded-error data via interval analysis," Math. Comput. Simulation, vol. 35, no. 4, pp. 1923-1937, 1993.
[29] P. Buschka, A. Saffiotti, and Z. Wasik, "Fuzzy landmark-based localization for a legged robot," in Proc. IEEE/RSJ Int. Conf. Intelligent Robots and Systems (IROS'O0), Takamatsu, Japan, 2000, pp. 1205-1210.

[30] M. Kieffer, L. Jaulin, E. Walter, and D. Meizel, "Guaranteed mobile robot tracking using interval analysis," in Proc. MISC'99: Workshop on Applications of Interval Analysis to Systems and Control, Girona, Spain, Feb. 1999, pp. 347-359.

\section{On Mobile Robot Localization From Landmark Bearings}

\author{
Ilan Shimshoni
}

Abstract-This paper deals with the problem of robot localization from noisy landmark bearings measured by the robot. We present a new localization method which is based on linear constraints, one due to each bearing measurement. This linear system can be solved at low computational cost but yields not very accurate results. Therefore, we transform the system to an equivalent linear system which yields virtually optimal results at a small fraction of the cost of a nonlinear optimization method, which usually achieves the optimal result. Experimental results showing the quality of the results and the low computational cost are presented.

\section{Index Terms-Angle measurements, landmarks, robot localization.}

\section{INTRODUCTION}

When mobile robots or automated guided vehicles (AGVs) are moving in their workspaces, one of the basic problems which needs to be solved is for the robot to know its position and orientation in the plane as accurately as possible. One of the standard methods for performing this task is to put landmarks in known locations in the workspace. In any place in the workspace, the robot is able to measure the bearings to a sufficient number of these landmarks. Using three or more such measurements, the robot is able to estimate its position and orientation in the plane [1]-[4].

The most widely used method for computing this estimate is a geometric method based on the idea that the angle between two such bearing measurements yields the constraint that the robot's position is limited to lie on a circle. Adding an additional bearing measurement yields two more circles whose intersection is the desired location. The problem begins when there are errors in the measurements. To overcome this problem, more than three landmarks are placed in the workspace, and all the measured bearings have to be used to get the optimal estimate for the robot's position. The geometric method does not lend itself naturally to more than three bearing measurements. Thus, costly nonlinear minimization techniques with their known problems have to be employed.

We would like, therefore, to take a different approach, which has been presented in [5], but without dealing with the accuracy problems.

Manuscript received January 16, 2001; revised December 16, 2001. This paper was recommended for publication by Associate Editor D. Pai and Editor S. Hutchinson upon evaluation of the reviewers' comments. This work was supported in part by the Israeli Ministry of Science under Grant 9766 and Grant 2104. This paper was presented in part at the International Conference on Robotics and Automation, Seoul, Korea, May, 2001.

The author is with the CAIP Center, Rutgers University, Piscataway, NJ 08854 USA, on leave from the Department of Industrial Engineering and Management, Technion, Technion City 32000, Israel (e-mail: ilans@ie.technion.ac.il).

Digital Object Identifier 10.1109/TRA.2002.805663 